\title{
REPONSES DE DEUX VARIETES DE SESAME A L'INOCULATION AVEC DES CHAMPIGNONS MYCORHIZIENS ARBUSCULAIRES CANDIDATS
}

\author{
M. DIOUF ${ }^{1}$, S. BOUREIMA ${ }^{1}$ et T. A. DIOP ${ }^{2}$ \\ ${ }^{1}$ Centre d'Etude Régional pour l'Amélioration de l'Adaptation à la Sécheresse, B.P. 3320, Thiès, Sénégal. \\ E-mail : boureimaseyni@yahoo.fr \\ 2Laboratoire de Biotechnologies des Champignons, Département de Biologie végétale, Faculté des Sciences \\ et Techniques, Université Cheikh Anta Diop, B.P. 5020, Dakar, Sénégal
}

\begin{abstract}
RESUME
La pauvreté des sols et les sécheresses récurrentes constituent un facteur limitant à la productivité du sésame au Sénégal. Pour contribuer à la résolution de ces problèmes, l'utilisation des champignons mycorhiziens arbusculaires est devenue une voie active de recherche. Cette étude a été réalisée pour évaluer les réponses de deux variétés de sésame à l'inoculation mycorhizienne arbusculaire. Deux expérimentations indépendantes ont été conduites en serre, suivant un dispositif en blocs complets randomisés avec 4 répétitions. Les résultats indiquent que les champignons MA favorisent l'augmentation du nombre de feuilles et de la surface foliaire. Chez Ceraas-1-98, la surface foliaire a augmenté de $45 \%$, pour les plantes inoculées avec la souche DAOM 197198 et le poids sec des racines de $121 \%$ avec la souche DAOM 227 130. Chez Primoca une augmentation de $100 \%$ a été notée pour la surface foliaire des plantes inoculées avec la souche DAOM 227131 et le poids sec des racines de $80 \%$, avec les isolats DAOM 227 131 et DAOM 197 198. Les isolats fongiques indigènes utilisés sont un moyen biologique d'améliorer la productivité du sésame, prometteur en zone semi-aride.
\end{abstract}

Mots clés : Mycorhize arbusculaire, sésame, Sénégal.

\begin{abstract}
RESPONSES OF TWO SESAME CULTIVARS TO INOCULATION WITH SOME ARBUSCULAR MYCORRHIZAL FUNGI CANDIDATES

Soil poverty and recurrent drought are important factors limiting sesame productivity in Senegal. In order to contribute to the resolution of these problems, the use of arbuscular mycorrhizae fungi (AMF) has become an active area of research worldwide. This study was carried out to evaluate the response of two sesame varieties to Abuscular mycorrhiza (AMF) inoculation. Two separates experiments were conducted in the greenhouse in a completely randomized blocks design, with 4 replications. Results show that AM enhanced leaves number and leaves area. Leaves area of Ceraas-1-98 was significantly increased by $45 \%$, when inoculated with DAOM 197198 isolate and root biomass increased by 121\%, when inoculated with DAOM 227 130. Primoca leaf area was increased by $100 \%$, when inoculated with DAOM 227131 and its root biomass increased by $80 \%$ when inoculated by either DAOM 227131 or DAOM 197 198. The use of soil-born mycorrhizal fungi, in this study, was a biological method to improve sesame productivity in a semi-arid area.
\end{abstract}

Keywords : Vesicular arbuscular mycorrhizae, sesame. 


\section{INTRODUCTION}

Dans la nature, la majorité des végétaux terrestres vit en symbiose avec les champignons mycorhiziens. Cette association est appelée mycorhize, du grec mukês pour champignon et rhiza pour racine (Harley and Smith, 1983). On distingue plusieurs types d'associations mycorhiziennes mais les mycorhizes arbusculaires (MA) constituent les symbioses végétales les plus répandues car elles existent chez plus de $90 \%$ des végétaux terrestres (Wang and Qiu, 2006). Les mycorhizes améliorent la structure du sol par la formation d'agrégats (Bethlenfalvay et Schüepp, 1994 ; Mathias et Daniel, 2006). En outre, ils augmentent la résistance des plantes aux maladies (Linderman, 1994), et favorisent la nutrition hydro-minérale ce qui réduit l'utilisation massive d'engrais minéraux préservant la qualité de l'environnement.

Par ailleurs, plusieurs auteurs (Medina et al., 1990 ; Subramanian and Charest, 1997 ; Duponnois et al., 2004) ont noté que l'utilisation des MA permet un accroissement du rendement en biomasse des plantes, d'accélérer la floraison (Abdoul-Nasr, 1996), de minimiser les effets de la sécheresse, du gel, et d'autres stress environnementaux (Dalpé, 1998 ; Meddich et al., 2004). Ceux-ci témoignent de toute l'importance de leur utilisation en agriculture durable.

Cependant, l'influence des mycorhizes arbusculaires sur la croissance du sésame ont fait l'objet de peu de travaux de recherches. Pourtant, le sésame fait partie des plus anciens oléagineux au monde et est très adapté aux régions arides et subarides pour ses modestes besoins en eau (Boureima, 2005). Les graines de sésame sont riches en huile $(50 \%)$, en protéines $(25 \%)$, en vitamines $(B, E)$ et en minéraux ( $\mathrm{Ca}, \mathrm{P}, \mathrm{Mg})$. Actuellement, sa culture présente un engouement dans le monde rural, mais les rendements sont limités à cause du faible niveau des intrants utilisés (faible pouvoir d'achat des paysans) et de la sensibilité de la plante aux conditions pédo-climatiques défavorables (Diouf, 2004). Il convient donc de mettre au point des solutions moins onéreuses, telles que l'inoculation MA, respectueuses de l'environnement et plus durables pour optimiser la nutrition hydro-minérale du sésame et par conséquent augmenter sa productivité.

L'objectif de ce présent travail est d'évaluer les réponses à l'inoculation MA par quatre isolats fongiques sur la croissance de deux variétés de sésame appartenant à deux types botaniques différents.

\section{MATERIEL ET METHODES}

Les expérimentations ont été conduites en serre au Centre d'étude régional pour l'amélioration de l'adaptation à la sécheresse (CERAAS) au Sénégal.

\section{MATERIEL VEGETAL}

\section{Les variétés de sésame}

Le matériel végétal est constitué de 2 variétés de sésame, Ceraas-1-98, pour le type botanique monotige et Primoca, pour le type botanique très ramifié. Les caractéristiques agronomiques de ces deux variétés sont données au Tableau 1.

Tableau 1 : Caractères agronomiques des variétés étudiées.

Agronomic characteristics of the varieties.

\begin{tabular}{lcccccc}
\hline \multirow{2}{*}{ Variétés } & $\begin{array}{c}\text { Cycle } \\
\text { (jours) }\end{array}$ & Type botanique & \multicolumn{2}{c}{ Rendement $\left(\mathrm{kg} \mathrm{ha}^{-1}\right)$} & Couleur de & P1000G $(\mathrm{g})$ \\
\cline { 5 - 6 } Ceraas-1-98 & 75 & Monotige & - & 625 & Blanche & 2,36 \\
\hline Primoca & $110-120$ & Très ramifié & 843 & 879 & Brune & 3,03 \\
\hline P1000G = Poids de mille graines & & & &
\end{tabular}




\section{Matériel fongique}

Les isolats fongiques utilisés proviennent de la collection du Laboratoire de Biotechnologies des Champignons (LBC) du département de Biologie Végétale de l'Université Cheikh Anta Diop de Dakar (Ucad). Ce sont : les souches DAOM 227 131 de Glomus mosseae, DAOM 227115 de Glomus verruculosum, DAOM 227130 de Glomus fasciculatum et DAOM 197198 de Glomus intraradices.

Pour la production de l'inoculum, ces isolats fongiques ont été multipliés séparément en serre dans des pots de 1,5 litre de capacité. Le maïs a été utilisé comme plante piège.

\section{MATERIEL CHIMIQUE}

Le matériel chimique est essentiellement constitué par : l'hypochlorite de sodium $(\mathrm{NaOCl}$, $1 \%$ ), l'eau distillée, engrais minéral N-P-K de formulation $15-15-15$, du KOH (10 \%) et du bleu de trypan $(0,05 \%)$.

\section{AUTRES MATERIELS}

Des pots en plastique $(30 \mathrm{~cm}$ de diamètre $x$ $28 \mathrm{~cm}$ de hauteur) et du sol de type "Dior" ont été utilisés comme supports. Pour les mesures, le scanner LC 4800, le logiciel Winfolia (Regent instrument inc, Canada), une étuve, une balance de précision $10^{-2} \mathrm{~g}$, un bain marie ont été utilisés.

\section{METHODE}

\section{Dispositifs expérimentaux}

Deux expérimentations ont été conduites en pots selon un dispositif en blocs complets randomisés avec 4 répétitions. Chaque unité expérimentale a été représentée par 2 pots.

Dans l'expérimentation 1 , qui a porté sur le type botanique monotige avec la variété Ceraas-1-98, le facteur "Inoculation" a été étudié à 4 niveaux : un témoin non inoculé et inoculation avec chacune des souches DAOM 227 131, DAOM 227130 et DAOM 197198.

Dans l'expérimentation 2 qui a porté sur le type botanique très ramifié Primoca, le facteur "Inoculation" a été étudié à 4 niveaux : un témoin, les souches DAOM 227 131, DAOM 227115 et DAOM 197198.

\section{Conduite de la culture}

Chaque pot en plastique est rempli avec $15 \mathrm{Kg}$ de sol de type «Dior» prélevé à une profondeur de 0 - $30 \mathrm{~cm}$ sur la parcelle expérimentale non cultivée du CERAAS au Centre National de Recherches Agronomiques (CNRA) de Bambey. Le sol utilisé est préalablement tamisé (diamètre $2 \mathrm{~mm}$ ) et autoclavé à $120^{\circ} \mathrm{C}$ pendant une heure/ jour sur deux jours consécutifs. Le tableau 2 présente la composition physico-chimique du sol utilisé.

Le sol dans les pots est arrosé à la capacité au champ la veille du semis. Pour l'inoculation, $15 \mathrm{~g}$ de substrat contenant des spores (40 spores/g), du sable et des fragments de racines mycorhyzées (fréquence $80 \%$ ) sont placés à $2 \mathrm{~cm}$ de profondeur puis recouvert du même sable. Le semis est effectué à raison de 5 graines préalablement traitées à l'hypochlorite de sodium $1 \%(\mathrm{NaOCl})$ pendant 3 minutes puis rincées abondamment avec de l'eau distillée stérilisée.

Les pots témoins non inoculés ont reçu chacun la même quantité d'inoculum stérilisé. La levée a été effective 4 jours après semis (jas) et un démariage est effectué à raison d'un pied par pot à 14 jas, suivi d'un apport de $40 \mathrm{~kg} / \mathrm{ha}$ d'engrais N-P-K (15-15-15) correspondant à $4 \mathrm{~g}$ par pot, sous une forme broyée. Pendant tout le cycle, les pots sont maintenus à la capacité au champ. 
Tableau 2 : Composition physico-chimique du sol utilisé.

Characteristics of the soil of study.
\begin{tabular}{lc}
\hline Composants & Teneur \\
\hline Sable & $94,8 \%$ \\
\hline Argile + limon & $5,8 \%$ \\
\hline Carbone total & $2,61 \%$ \\
\hline Azote total & $0,29 \% 0$ \\
\hline C/N ratio & 9,2 \\
\hline Potassium total $(\mathrm{meq} / 100 \mathrm{~g})$ & 0,034 \\
\hline Phosphore total $(\mathrm{ppm})$ & 195 \\
\hline Phosphore disponible $(\mathrm{ppm})$ & 26,77 \\
\hline Calcium $(\mathrm{meq} / 100 \mathrm{~g})$ & 1,1 \\
\hline Magnésium $(\mathrm{meq} / 100 \mathrm{~g})$ & 0,47 \\
\hline $\mathrm{pH}($ sol/eau ratio $1: 2)$ & 5,2 \\
\hline $\mathrm{pH}($ sol/ KCl ratio $1: 2)$ & 4,3 \\
\hline Humidité à pF 4,2 & $1,67 \%$ \\
\hline
\end{tabular}

\section{Paramètres mesurés}

La croissance des plantes a été suivie une fois par semaine au cours du cycle et a porté sur le nombre de feuilles sur pied (NF) et la hauteur des plantes (HP).

Dès l'apparition du premier bouton floral, une des deux plantes de chaque unité expérimentale a été coupée pour déterminer la surface foliaire à l'aide du scanner LC 4800 et du logiciel Winfolia (Regent instrument inc, Canada). A maturité, les plantes ont été coupées puis la biomasse sèche déterminée après séchage à l'étuve à $65{ }^{\circ} \mathrm{C}$ jusqu'à poids constant. Les racines ont été ensuite soigneusement récupérées sur un tamis après un léger jet d'eau puis séchées à l'étuve à $65^{\circ} \mathrm{C}$ pendant 3 jours et le poids sec déterminé à l'aide d'une balance de précision $10^{-2} \mathrm{~g}$.

Pour évaluer le taux de la mycorhization, un échantillon de fines racines a été prélevé sur chaque plant récolté. Ces racines ont été soigneusement rincées à l'eau de robinet pour éliminer les particules de sable puis éclaircies dans des tubes à essai contenant du $\mathrm{KOH}$ $(10 \%)$ à $95^{\circ} \mathrm{C}$ pendant une heure. Elles ont été ensuite abondamment rincées à l'eau de robinet pour éliminer le $\mathrm{KOH}$ puis portées au bain marie à $95^{\circ} \mathrm{C}$ pendant 30 minutes dans un mélange de bleu de trypan $(0,05 \%)$. Le taux de mycorhization des plantes a été déterminé par la méthode de Gridline intersect method (Giovannetti and Mosse, 1980).
Les données mesurées ont été soumises à une analyse de variance en utilisant la procédure GLM du logiciel SAS (SAS Institute Inc., Cary, NC, USA, 2006). Le test de Newman-Keuls a été utilisé pour la comparaison des moyennes au seuil de $5 \%$.

\section{RESULTATS}

\section{EXPERIMENTATION 1}

Le suivi du rythme d'apparition des feuilles a montré que l'inoculation MA a permis la formation d'un plus grand nombre de feuilles par rapport au témoin.

La figure 1 présente l'évolution du nombre de feuilles de la monotige Ceraas-1-98 suivant le type des isolats fongiques. On note une phase d'émission lente des feuilles jusqu'à 31 jas suivie d'une phase d'accélération allant de 31 à 66 jas puis une phase stationnaire vers la fin du cycle.

Les plantes inoculées ont présenté plus de feuilles que les témoins à partir du $45^{\mathrm{e}}$ jas et ce jusqu'à la fin du cycle où l'on a enregistré 46 feuilles/plante chez les plantes mycorhizées contre 34 chez les plantes non mycorhizées.

L'inoculation a aussi eu comme effet d'accroître significativement la surface foliaire des plantes. La souche DAOM 197198 a augmenté la surface foliaire de $45 \%$. Les souches DAOM 227130 et 227131 sont similaires avec un accrois- 
sement moyen de $23 \%$ comparés au témoin (Figure 2). Par contre la hauteur des plantes n'a pas été influencée par la mycorhization et ce quel que soit l'isolat fongique (Figure 3 ).

La biomasse aérienne sèche a été accrue de manière significative par l'inoculation MA. Ainsi, la souche DAOM 227130 a permis l'obtention d'une biomasse aérienne plus importante avec un accroissement de $73 \%$. Les plantes inoculées avec les souches DAOM 197198 et 227131 sont similaires et ne sont pas significativement différentes du témoin (Figure 4).

L'analyse statistique a aussi révélé une influence significative de l'inoculation mycorhizienne arbusculaire sur le poids sec des racines. L'isolat fongique DAOM 227130 a induit un accroissement du poids sec des racines de $121 \%$. Par contre les plantes inoculées avec les souches DAOM 197198 et 227131 ne sont pas significativement différentes du témoin (Figure 5). Par ailleurs, la souche DAOM 227 130 a présenté le meilleur taux de colonisation des racines. Les souches DAOM 227131 et DAOM 197198 ont présenté des taux similaires et inférieurs (Tableau 3).

D'une manière générale le taux de colonisation des racines est faible pour tous les isolats fongiques.

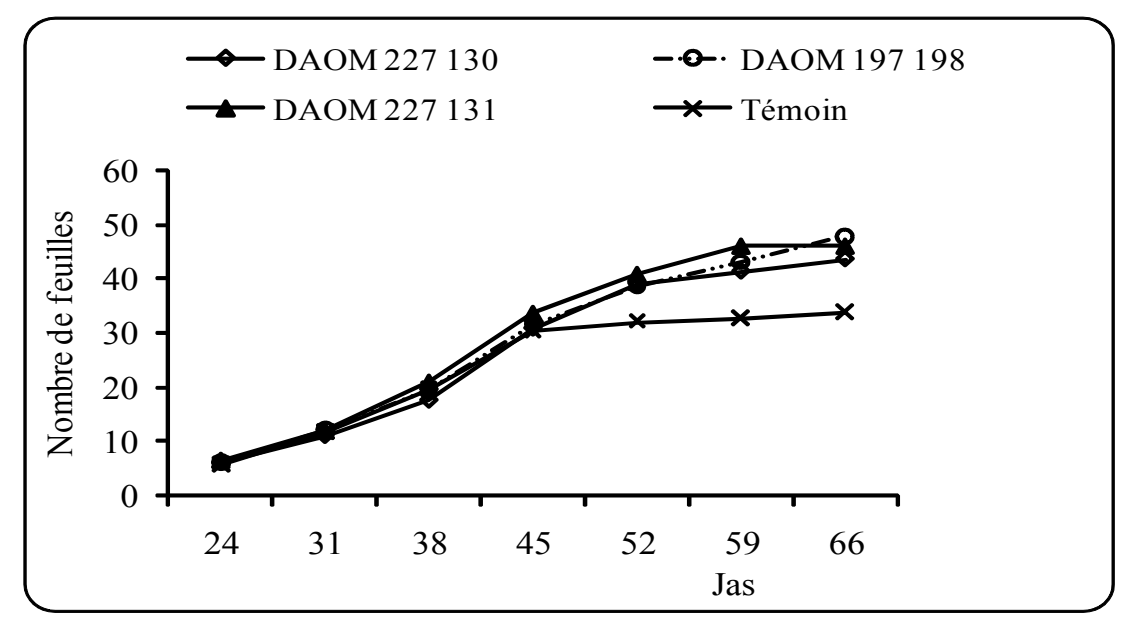

Figure 1 : Effets de l'inoculation sur le nombre de feuilles de Ceraas-1-98.

Effects of inoculation on number of leaves of Ceraas-1-98.

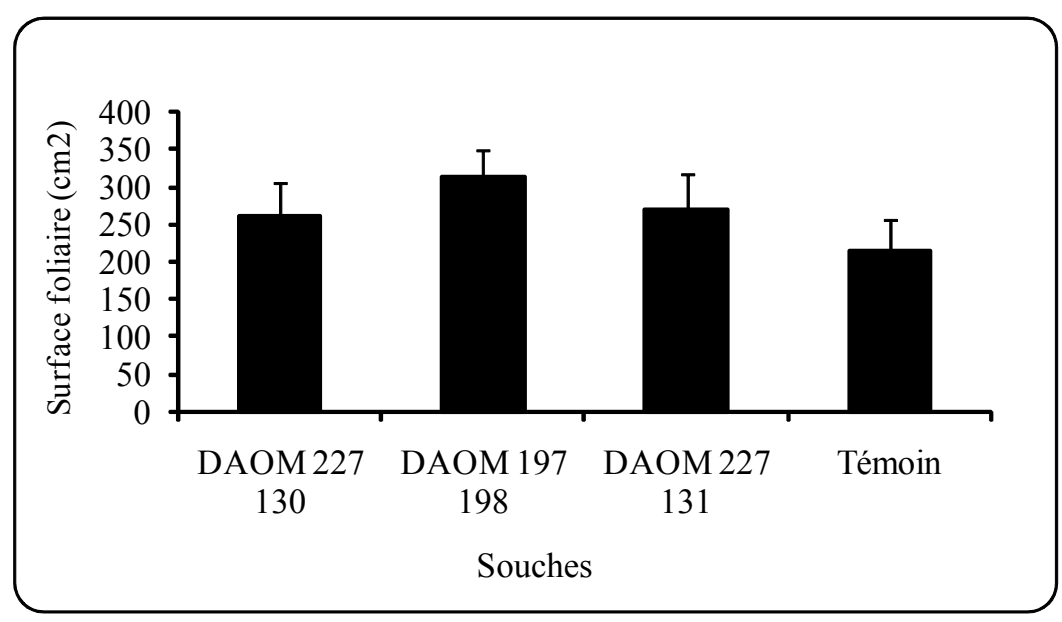

Figure 2 : Effets de l'inoculation sur la surface foliaire de Ceraas-1-98.

Effects of inoculation on the leaf area of Ceraas-1-98. 


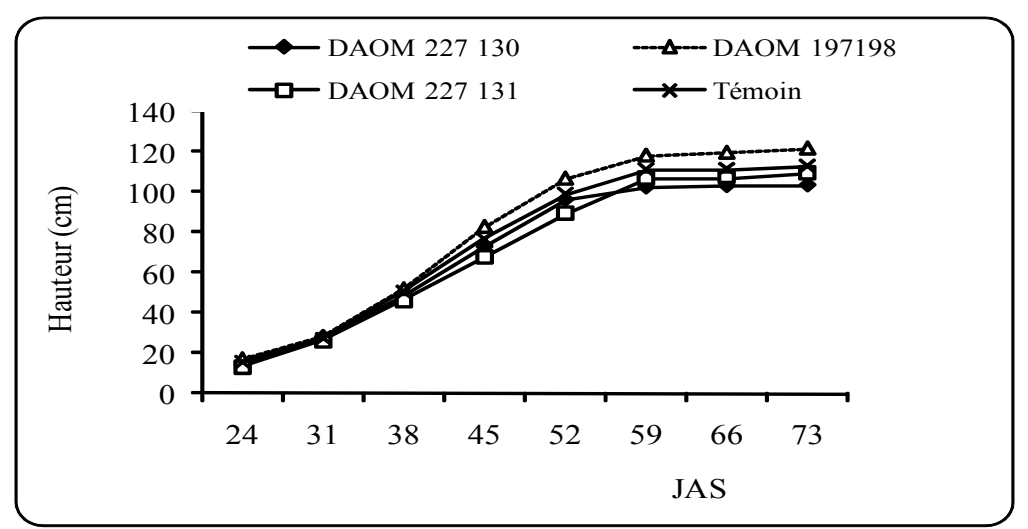

Figure 3 : Hauteur de Ceraas-1-98 selon les souches.

Ceraas-1-98 plants high relate to isolates.

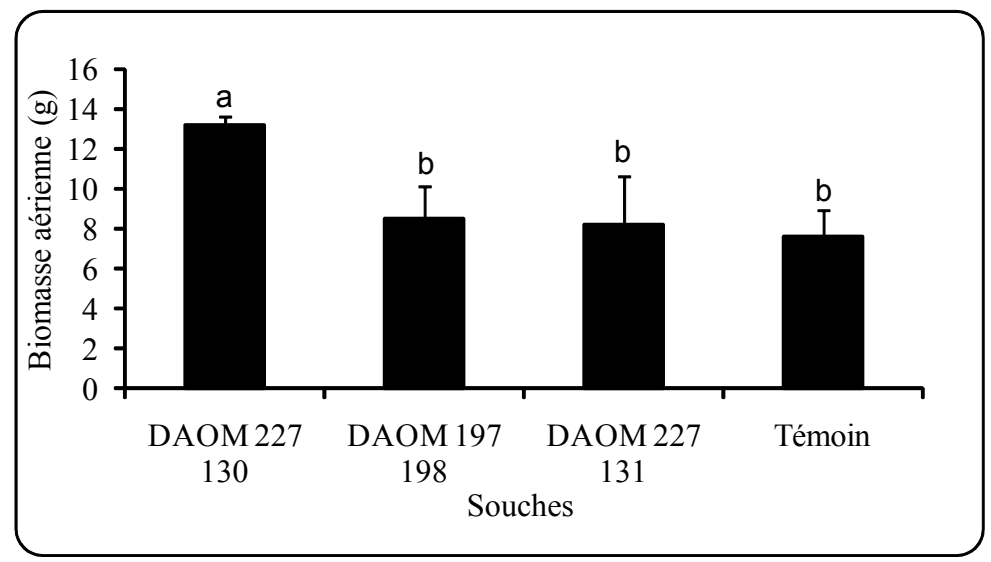

Figure 4 : Biomasse aérienne de Ceraas-1-98 selon les isolats fongiques. Air biomass of Ceraas-1-98 relate to isolates.

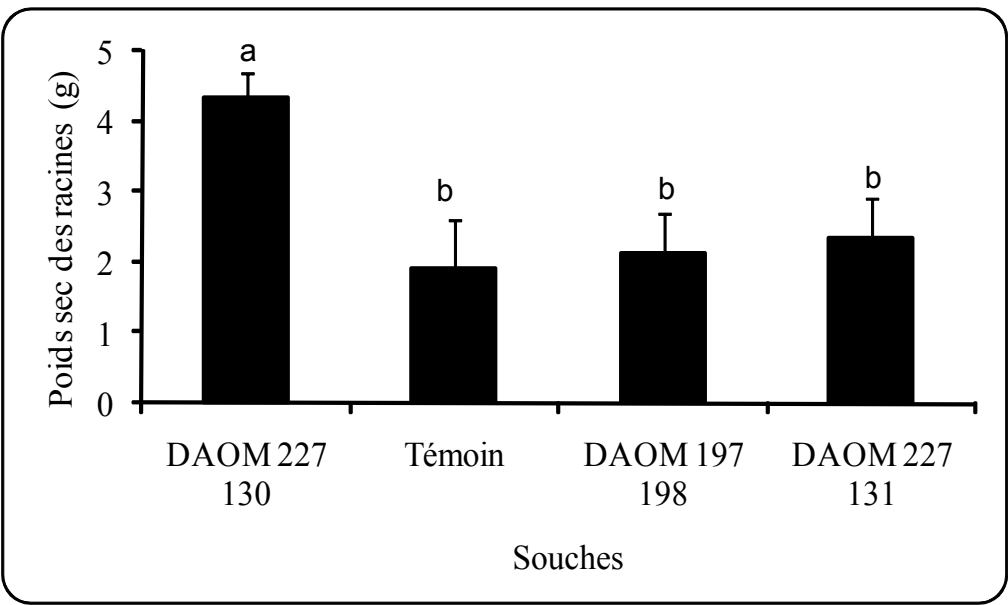

Figure 5 : Effets de l'inoculation sur la biomasse racinaire de Ceraas-1-98.

Effects of inoculation on root biomass of Ceraas-1-98. 
Tableau 3 : Taux de mycorhization des deux variétés.

Mycorrization rate of the varieties.

\begin{tabular}{lcc}
\hline Variétés & Isolats & Taux de mycorhization (\%) \\
\hline \multirow{4}{*}{ Ceraas-1-98 } & Témoin & $0 \mathrm{c}$ \\
& DAOM 227 131 & $20,70 \mathrm{~b}$ \\
& DAOM 227 130 & $37,74 \mathrm{a}$ \\
& DAOM 197 198 & $17,91 \mathrm{~b}$ \\
\hline \multirow{3}{*}{ Primoca } & Témoin & $0 \mathrm{c}$ \\
& DAOM 227 131 & $24,75 \mathrm{a}$ \\
& DAOM 197 198 & $21,68 \mathrm{a}$ \\
\hline
\end{tabular}

\section{EXPERIMENTATION2}

Au niveau de la deuxième expérimentation, qui a porté sur la variété Primoca, l'inoculation MA a eu également comme effet, l'augmentation du nombre de feuilles qui est passé de 98 chez les témoins à 125 feuilles/plante en moyenne chez les pieds inoculés (Figure 6). La même dynamique d'apparition du nombre de feuilles a été observée comme chez la monotige, un rythme d'apparition plus lent jusqu'à 36 jas, suivi d'une phase d'accélération jusqu'à 66 jas puis une phase stationnaire.

De même, la surface foliaire a été significativement accrue de $100 \%$ particulièrement chez les plantes inoculées avec la souche DAOM 227 131. Les souches DAOM 197198 et 227115 ont augmenté la surface foliaire respectivement de $57,67 \%$ et $31 \%$ (Figure 7). La hauteur des plantes n'a par contre, pas été influencée par la mycorhization et ce quel que soit l'isolat fongique (Figure 8).

D'une manière générale, tous les isolats fongiques ont significativement augmenté la biomasse aérienne sèche. Mais ce sont surtout les souches DAOM 227131 et DAOM 227115 qui ont permis d'obtenir un accroissement de la biomasse de $43 \%$ (Figure 9 ).

L'effet de l'inoculation sur le poids sec des racines a été significatif. Les isolats fongiques DAOM 227131 et DAOM 197198 sont similaires et ont accrue le poids sec des racines de $80 \%$ en moyenne (Figure 10).

Ces deux isolats fongiques ont aussi présenté les taux de colonisation les plus élevés comparativement à l'isolat DAOM 227115 (Tableau 3) même si en général le pourcentage de colonisation des racines a été faible chez tous les isolats fongiques.

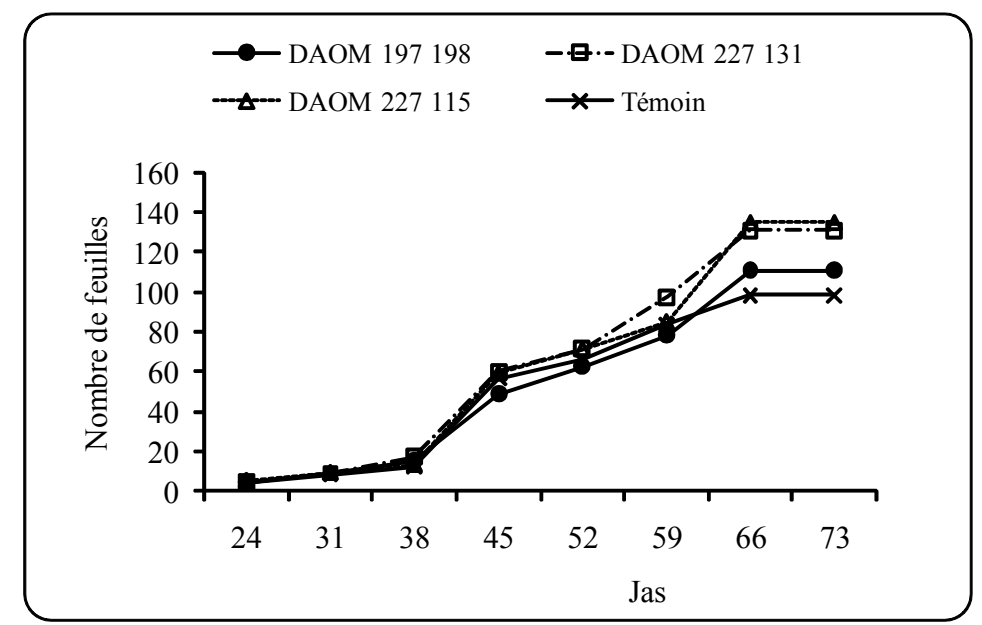

Figure 6 : Effets de l'inoculation sur le nombre de feuilles de Primoca.

Effects of inoculation on number of leaves of Primoca. 


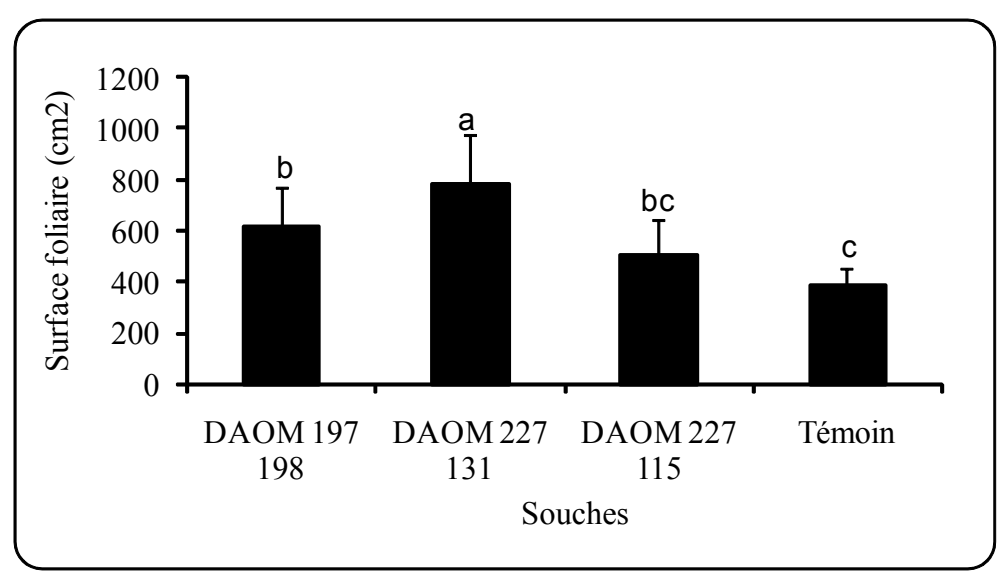

Figure 7 : Effets de l'inoculation sur la surface foliaire de Primoca.

Effects of inoculation on the leaf area of Primoca.

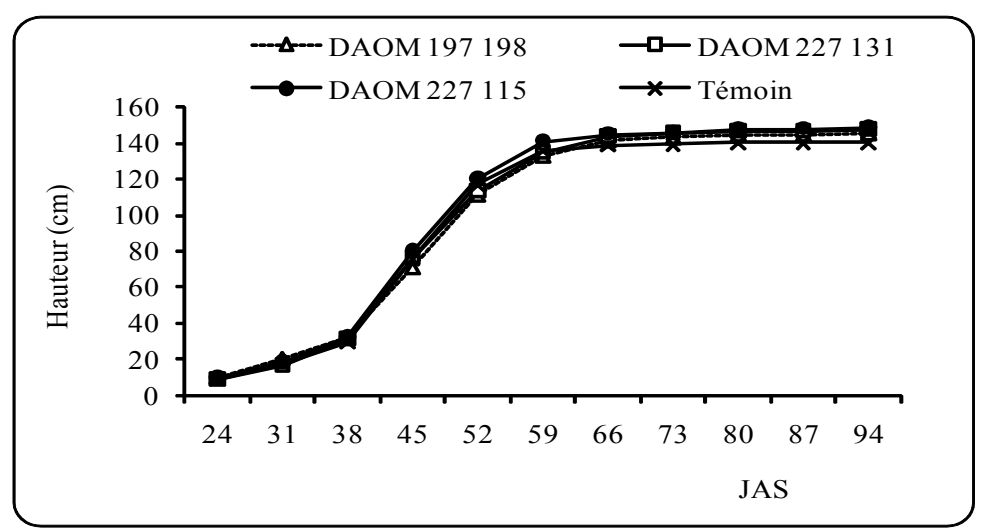

Figure 8 : Hauteur de Primoca en fonction des souches.

Primoca's heigh relating to isolates.

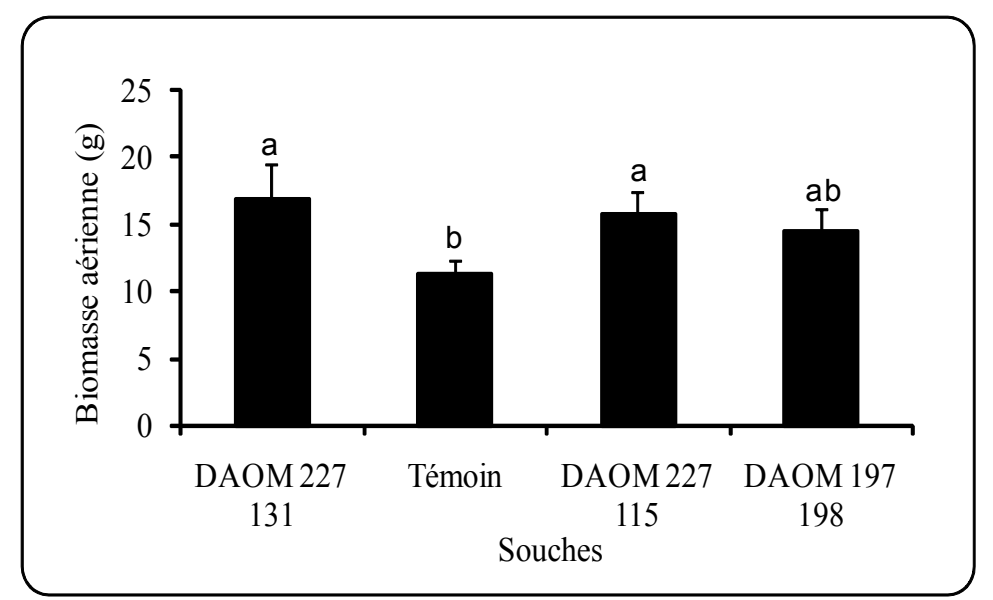

Figure 9 : Effets de l'inoculation sur la biomasse aérienne de Primoca.

Effects of inoculation on the air biomass of Primoca. 


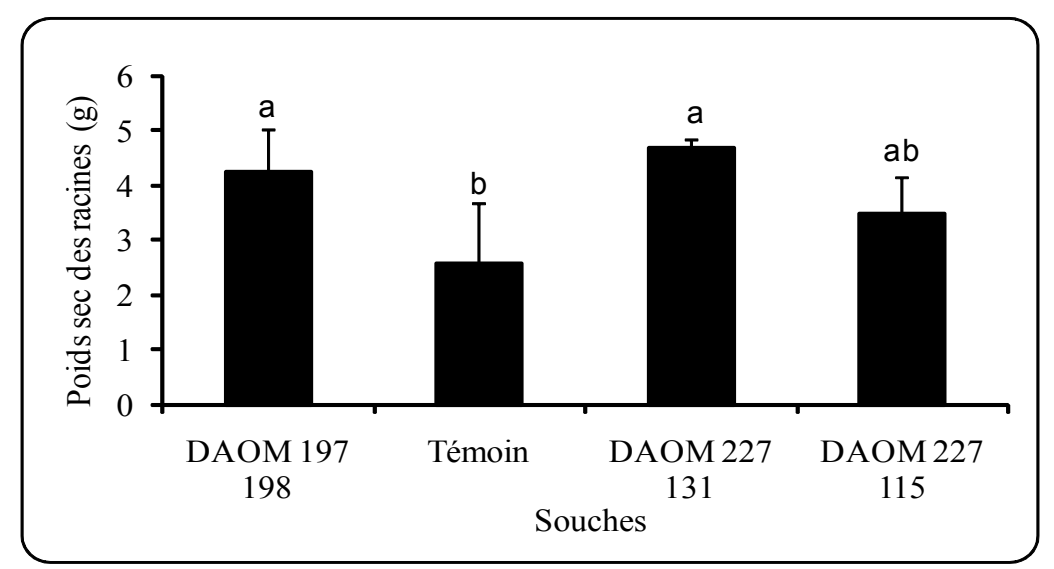

Figure 10 : Effets de l'inoculation sur la biomasse racinaire de Primoca.

Effects of inoculation on the root biomass of Primoca.

\section{DISCUSSION}

Les résultats indiquent que les champignons mycorhiziens arbusculaires (MA) ont une influence significative sur la croissance du sésame. Cependant, le degré de réponse à l'inoculation MA dépend de la variété et de la souche fongique. Ces résultats corroborent ceux de Kumar et al. (1998). L'accroissement du nombre de feuilles et/ou de la surface foliaire par l'inoculation MA a été rapportée chez le maïs (Kothari et al., 1990) et la laitue (Tobar et al., 1994). L'augmentation de la surface foliaire chez les plantes mycorhizées serait le résultat de l'amélioration de leur capacité à acquérir l'azote $\left(\mathrm{NO}_{3}^{-}\right)$du sol via les hyphes extra-matriciels des champignons MA associés (Tobar et al., 1994). Cet accroissement de la surface foliaire par les champignons MA est très bénéfique pour le maintien d'un taux photosynthétique élevé (Augé et al., 1987 ; Panwar, 1993), particulièrement chez le sésame où la croissance des structures reproductives est plus basée sur la photosynthèse courante que par une remobilisation de la matière sèche (Narayanan and Balakrishna, 1982).

La mycorhization accroit significativement le poids sec des racines. En effet, les champignons MA améliorent l'assimilation du phosphore par les plantes auxquelles ils sont associés en favorisant l'exploration physique des espaces interstitielles du sol par leurs hyphes (O'Keefe and Sylvia, 1993). C'est probablement cette biodisponibilité du phosphore qui a contribué au propre développement des racines et par conséquent, à l'augmentation du poids sec. Dans nos conditions arides où le stress hydrique est fréquent au cours du cycle cultural, l'accroissement de la biomasse aérienne et racinaire contribue à l'amélioration du rendement en grain car celui-ci est directement fonction de la biomasse produite et de l'indice de récolte en condition de stress hydrique (Banziger et al., 2000). En outre, selon Yadav et al. (1997), il est évident qu'une augmentation de la masse racinaire permet d'obtenir des rendements élevés en condition de stress hydrique.

Généralement, le pourcentage de colonisation des racines par les isolats fongiques utilisés a été faible. Néanmoins, Guissou et al. (2001) ont noté que l'importance du taux de colonisation des racines ne préjuge pas de son efficacité sur la croissance des plantes.

\section{CONCLUSION}

Cette étude a permis de distinguer les isolats fongiques de par leur capacité à améliorer la croissance et le développement du sésame. Les résultats obtenus ont permis de montrer que l'utilisation de certains isolats fongiques mycorhiziens permet d'améliorer significativement la surface foliaire, les biomasses aérienne et racinaire et par conséquent la productivité chez le sésame. Les isolats fongiques utilisés, étant indigènes et donc adaptés aux conditions locales, pourraient constituer un moyen biologique prometteur pour l'amélioration de la production du sésame au Sénégal. Cependant, il se dégage des résultats, 
que l'efficacité de la symbiose dépend de la bonne combinaison isolat fongique/variété. Une détermination du meilleur couple symbiotique entre les souches et les variétés devrait être donc un préalable. Nous avons également noté une tendance positive entre le taux de colonisation des racines et l'efficacité de la souche fongique.

\section{REFERENCES}

Aboul-Nasr A. 1996. Effects of vesicular-arbuscular mycorrhiza on Tagetes erecta and Zirinis elegans. Mycorrhiza 6 : 61 - 64 .

Augé R. M., Schekel K. A. and R. L. Wample. 1987. Rose leaf elasticity changes in response to mycorrhizal colonisation and drought acclimation. Physiologia Plantarum 70 : $175-182$.

Bänziger M., Edmeades G. O. and M. Bellon. 2000. Breeding for drought and nitrogen stress tolerance in maize: from theory to practice. Mexico, D. F., Centro Internacioncal de Mejoramiento de Maíz y Trigo, 68 p.

Bethlenfalvay G. J. and H. Schüepp. 1994. Arbuscular mycorrhizas and agrosystem stability. In: S. Gianinazzi and H. Schüep (Eds.). Impact of arbuscular mycorrhizas on sustainable agriculture and natural ecosystems. ALS, Birkhauser, Basel : pp 117 - 131.

Boureima S. 2005. Besoins en eau, croissance et productivité du type botanique ramifié du sésame (Sesamum indicum L.) en zone semi-aride du sénégal. Mém. ingénieur, Ecole Nationale Supérieure d'Agriculture, Thiès, Sénégal, $60 \mathrm{p}$.

Dalpé Y. 1998. Biodiversity of mycorrhizal fungi. Electronic publication prepared for the Review of biodiversity in the Canadian agricultural soils. http: www.res2.agr.ca

Diouf M. 2004. Programme de formation et de recherche-développement pour la relance de la filière sésame (Sesamum indicum L.) au Sénégal. Document de projet, ISRACERAAS, Thiès, $21 \mathrm{p}$.

Duponnois R., Colombet A., Hien V. and J. Thioulouse. 2004. The mycorrhizal fungus Glomus intraradices and rock phosphate amendment influence plant growth and microbial activity in the rhizosphere of Acacia holosericea. Soil Biology and Biochemistry, Elsevier, 37 : 1460 - 1468
Giovannetti M. and B. Mosse. 1980. An evaluation of techniques for measuring vesicular arbuscular mycorrhizal infection in roots. New Phytol. 84 : 489 - 500.

Guissou T., Bâ A. M., Plenchette C., Guinko S. et R. Duponnois. 2001. Effets des mycorhizes à arbuscules sur la tolérance à un stress hydrique de quatre arbres fruitiers: Balanites aegyptiaca (L.) Del., Parkia biglobosa (jacq.) Benth., Tamarindus indica L. et Zizyphus mauritiana Lam. Sécheresse $12(2)$ : $121-127$.

Harley J. L. and S. E. Smith. 1983. Mycorrhizal symbiosis. Academic Press, New York and London UK, 483 p.

Kothari S. K., Marschner H. and E. George. 1990. Effect of VA mycorrhizal fungi and rhizosphere microorganisms on root and shoot morphology, growth and water relations in maize. New Phytol. 116 : $303-311$.

Kumar P. P., Shailja K. M., Rao M. S. and S. Ram Reddy. 1998. Genotype dependent variation in VAM infection and growth response of tender cultivars of sesame (sesamum indicum L.). J. Indian Bot. Soc. 77: 71 - 74.

Linderman R. G. 1994. Role of VAM fungi in biocontrol. In: F. L. Pfleger and R.G. Linderman (Eds.). Mycorrhizae and plant health. APS Press, St. Paul, Minn. : pp 1 - 26.

Mathias C. R. and L. M. Daniel. 2006. Mycorrhizas and soil structure. New Phytologist 171 : $41-53$

Meddich A., Oihabi A., Bizid E. et I. El hadrami. 2004. Rôle des champignons mycorhiziens VA dans la tolérance du palmier dattier (phoenix dactylifera) au déficit hydrique. Revue des régions arides $2: 640$ - 646 .

Medina O. A., Kretschmer J. A. E. and D. M. Sylvia. 1990. Growth response of field-grown Siratro (Macroptilium atropurpureum Urb.) and Aeschynomene american L. to inoculation with selected vesiculararbuscular mycorrhizal fungi. Biology and fertility of soil $9(1): 54-60$

Narayanan A. and K. Balakrishna Reddy. 1982. Growth, developpement and yield of sesame (Sesamum indicum) cultivars. Field Crops Research 5 (3) : 217 - 224.

O'keefe D. M. and D. M. Sylvia. 1993. Seasonal dynamics of the association between sweet potato and vesicular arbuscular mycorrhizal fungi. Mycorrhiza 3 : 115 - 122. 
Panwar J. D. S. 1993. Effect of VAM and Azospirillum on growth and yield of wheat. Indian J. Plant Physiol. 34 : 357 - 361.

Subramanian K. S. and C. Charest. 1997. Nutritional growth, and reproductive responses of maize (Zea mays L.) to arbuscular mycorrhizal inoculation during and after drought stress at tasseling. Mycorrhiza 7 (1) : 25 - 32.

Tarafdar J. C. and H. Marschner. 1994. Efficiency of VAM hyphae in utilisation of organic phosphorus by wheat plants. Soil Science and Plant Nutrition $40: 593$ - 600.
Tobar R., Azcon R. and J. M. Barea. 1994. The improvement of plant $\mathrm{N}$ acquisition from an ammonium-treated, drought-stressed soil by the fungal symbiot in arbuscular mycorrhizae. Mycorrhizae 4 : 105 - 108.

Wang B. and Y.-L. Qiu. 2006. Phylogenetic distribution and evolution of mycorrhizas in land plants. Mycorrhiza, 16 (5) : 299 - 363.

Yadav R., Courtois B., Huang N. and G. McLaren. 1997. Mapping genes controlling root morphology and root distribution in a doublehaploid population of rice. Theor. Appl. Genet. $94: 619$ - 632 . 